\section{Trans-scleral diode laser cyclophoto- coagulation in the treatment of diabetic neovascular glaucoma}

S Nabili and CM Kirkness
The Tennent Institute of Ophthalmology University of Glasgow Gartnavel General Hospital Glasgow, UK

Correspondence:

S Nabili

The Tennent Institute of Ophthalmology Gartnavel General Hospital Great Western Road Glasgow G12 OYN, UK Tel: +441412111039 E-mail: nabili@ hotmail.com

Received: 15 January 2003 Accepted in revised form: 6 May 2003

\begin{abstract}
Aims To assess efficacy of trans-scleral diode laser cyclophotocoagulation in the treatment of diabetic neovascular glaucoma refractory to medical therapy.

Methods Case notes of 20 eyes of 20 patients who had the treatment were analysed. The mean follow-up after initial treatment was $\mathbf{2 2 . 5}$ months (range of 18-24).

Results Mean (SD) pretreatment intraocular pressure (IOP) for the 20 eyes was $34.4 \mathrm{mmHg}$ (9.5) reducing to $18.2 \mathrm{mmHg}(12.4)$ at the final index visit $(P=0.0001)$. The mean (SD) number of topical antiglaucoma medication was significantly lowered from $3.9(0.3)$ to 1.2 (1.3). Four patients had visual acuity of $6 / 60$ or better before the treatment. Two of them maintained the same level of vision and the other two had their vision reduced over the course of study; however, none of them deteriorated beyond $6 / 60$. Six out of the remaining 16 patients who had vision of counting fingers or worse before treatment progressed to no perception of light at the final index visit. The mean (SD) number of treatment sessions was 1.45 (0.68). A total of 10 patients had previous pars plana vitrectomy (PPV). Patients with two or more PPVs developed hypotony (IOP $\leq 5)$. There were five eyes with hypotony, one of which became phthisical.

Conclusion Trans-scleral diode laser cyclophotocoagulation is a useful tool in the management of diabetic neovascular glaucoma. The current treatment regime needs to be adjusted to reflect higher risk of hypotony and phthisis in diabetic neovascular glaucoma in eyes that have had multiple pars plana vitrectomies.

Eye (2004) 18, 352-356. doi:10.1038/

sj.eye. 6700644
\end{abstract}

Keywords: cyclophotocoagulation; neovascular glaucoma; diabetes; refractory glaucoma; diode laser

\section{Introduction}

Coats was first to described rubeosis iridis in 1906. Since then, many causes of neovascularisation of the iris and angle have been described, but $97 \%$ of iris neovascularisation is associated with retinal ischaemia ${ }^{1}$ such as diabetic retinopathy. Neovascular glaucoma occurs when fibrovascular tissue proliferates on to the chamber angle, obstructs the trabecular meshwork, and consequently causes increased intraocular pressure (IOP). ${ }^{2}$ The key aspect in management remains early detection of iris rubeosis and treatment of underlying ischaemia. A nondilated slit-lamp examination including gonioscopy is essential for early detection. Adequate panretinal photocoagulation should be performed at the first sign of rubeosis or retinal neovascularisation. This will cause regression of new vessels in $68 \%$ of patients and normalisation of IOP in $42 \%$ of patients. ${ }^{3}$

Despite early treatment, in a significant percentage of patients, the IOP will remain elevated and the successful management of the disease may be very difficult and frequently results in complete loss of vision. Coagulation or destruction of the ciliary body to reduce the rate of production of aqueous and hence reduce IOP was first described in 1930 by Vogt. ${ }^{4}$ As technology has progressed, more sophisticated methods of cyclodestruction have been used. ${ }^{5}$ The main challenge has been to achieve safety and efficacy together. Potential complications seen with all modes of cyclodestruction include prolonged ocular inflammation, hypotony, 
phthisis bulbi, visual loss, postoperative pain, and intraocular haemorrhage.

Trans-scleral diode laser cyclophotocoagulation may offer improved safety over other forms of cyclodestruction. ${ }^{6-8}$ Its use in the treatment of diabetic neovascular glaucoma has not, however, been fully investigated. Conventional filtration surgery carries significant risk of complications in these patients and, therefore, treatments such as diode laser cyclophotocoagulation may offer an alternative.

We describe our experience in using diode laser cyclophotocoagulation in the treatment of diabetic neovascular glaucoma refractory to medical treatment. We report our results and caution against its use in eyes that have had multiple pars plana vitrectomies (PPVs) under current treatment protocol.

\section{Methods}

This is a consecutive case series study of 20 eyes that were treated between 1999 and 2001. These cases were identified retrospectively from a procedure log book. Their case notes were analysed. The mean follow-up was 22.5 months (range 18-24). The reasons for the treatment were

(a) risk of filtration surgery was deemed unacceptable;

(b) the patient refused surgical intervention.

Prior to diode laser cyclophotocoagulation, patients received local anaesthesia by subtenon injection. There were no recorded complications of subtenon local anaesthesia. Cyclophotocoagulation was performed using Oculight SX semiconductor 810nm diode laser (Iris medical instruments) and contact $\mathrm{G}$ probe (Iris medical instruments). Transillumination was used when the position of ciliary body was in doubt. A quartz fibre optic that protrudes $0.7 \mathrm{~mm}$ from the surface of a curved foot plate resting on the surgical limbus delivers the laser light $1.2 \mathrm{~mm}$ behind the limbus. A standard treatment protocol was used as advocated by Spencer and vernon. ${ }^{9}$ A total of $75 \%$ of the circumference of the globe was treated in each treatment session. This usually resulted in 15 applications. Energy used was $2.0 \mathrm{~W}$ for 2.0 s resulting in a total of $56 \mathrm{~J}$ in each treatment session. Patient case notes were reviewed. Age, sex, IOP pretreatment, at 1-2 weeks, 1, 3, 6, 12, 18, 24 months, visual acuities, topical and oral antiglaucoma medication, number of treatment sessions, and number of PPVs and complications were recorded. Statistical analysis was performed using computer software (Minitab v13). A two-tailed paired Student's $t$ test was used when data approximated to normal distribution and Mann-Whitney U-test when it did not. $\chi^{2}$ test was used to evaluate the relation between variables.

\section{Results}

A total of 20 eyes of 20 patients with diabetic neovascular glaucoma were treated. There were 11 males and nine females.

\section{Control of IOP (Figures 1 and 2) (Table 1)}

Mean (SD) pretreatment IOP was $34.4 \mathrm{mmHg}$ (9.5) Figures 1 and 2. This was reduced to $18.2 \mathrm{mmHg}$ (12.4) at the final index visit $(P=0.0001)$ Table 1 . The mean percentage reduction of IOP was $45 \%$. All patients had pretreatment IOPs of more than $22 \mathrm{mmHg}$. A total of $60 \%$ had IOPs of less than $22 \mathrm{mmHg}$ at the final index visit. Only one patient had a higher IOP at the final index visit than pretreatment.

\section{Usage of antiglaucoma medication}

Prior to treatment, patients were using a mean (SD) of 3.9. (0.3) different topical antiglaucoma medications. This

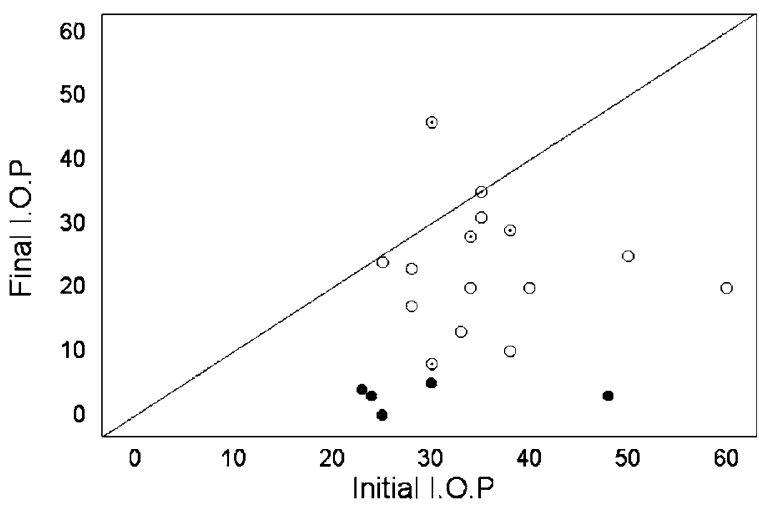

Figure 1 Scatter plot of final vs initial IOP (mmHg). Diagonal line is the line of equivalence. Empty circle indicates nonvitrectomised eyes. Dot circle indicates eyes with one PPV and solid circle indicates eyes with multiple PPVs.

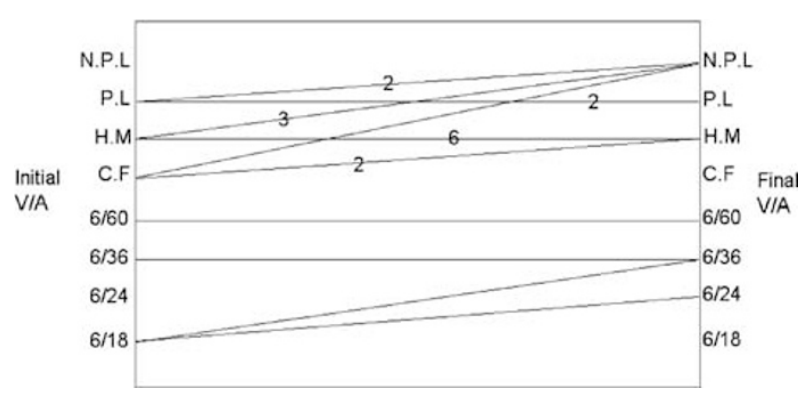

Figure 2 Mean IOP ( $\mathrm{y}$ axis) against time after first treatment ( $\mathrm{x}$ axis). The solid line is plotted in a standard way, excluding hypotonous eyes from follow-up after they become hypotonous. The dash line is replotted with continued inclusion of all patients. 
Table 1 A tabular presentation of key data allowing comparison between vitrectomised and nonvitrectomised eyes

\begin{tabular}{|c|c|c|c|c|c|c|}
\hline & Initial IOP & Fianl IOP & Initial $V / A$ & Final $V / A$ & Number of treatments & Number of PPVs \\
\hline 1 & 38 & 10 & $6 / 18$ & $6 / 24$ & 2 & 0 \\
\hline 2 & 60 & 20 & PL & PL & 2 & 0 \\
\hline 3 & 28 & 17 & $6 / 36$ & $6 / 36$ & 2 & 0 \\
\hline 4 & 35 & 31 & $\mathrm{HM}$ & $\mathrm{HM}$ & 1 & 0 \\
\hline 5 & 34 & 20 & $6 / 60$ & $6 / 60$ & 1 & 0 \\
\hline 6 & 25 & 24 & $\mathrm{HM}$ & $\mathrm{HM}$ & 1 & 0 \\
\hline 7 & 28 & 23 & $\mathrm{HM}$ & $\mathrm{HM}$ & 1 & 0 \\
\hline 8 & 40 & 20 & CF & HM & 2 & 0 \\
\hline 9 & 50 & 25 & $\mathrm{HM}$ & HM & 2 & 0 \\
\hline 10 & 33 & 13 & PL & PL & 1 & 0 \\
\hline 11 & 30 & 8 & $\mathrm{HM}$ & $\mathrm{HM}$ & 3 & 1 \\
\hline 12 & 35 & 35 & $6 / 18$ & $6 / 36$ & 1 & 1 \\
\hline 13 & 34 & 28 & $\mathrm{HM}$ & $\mathrm{HM}$ & 1 & 1 \\
\hline 14 & 38 & 29 & CF & $\mathrm{HM}$ & 1 & 1 \\
\hline 15 & 30 & 46 & $\mathrm{HM}$ & NPL & 1 & 1 \\
\hline 16 & 48 & 3 & HM & NPL & 1 & 2 \\
\hline 17 & 23 & 4 & $\mathrm{HM}$ & NPL & 1 & 2 \\
\hline 18 & 24 & 3 & PL & NPL & 1 & 2 \\
\hline 19 & 30 & 5 & $\mathrm{CF}$ & NPL & 1 & 3 \\
\hline 20 & 25 & 0 & PL & NPL & 1 & 3 \\
\hline
\end{tabular}

The eye presented at row 20 became phthisical.

PL: perception of light; CF: counting finger; NPL: no perception of light; HM: hand movement.

was reduced to a mean (SD) of $1.2(1.3)$ at the final index visit $(P=0.0001)$. Each active agent of topical medication was counted as one, for example brimonidine tartate was counted as one and Cosopt ${ }^{\mathbb{R}}$ as two. In all, 11 patients (55\%) needed topical antiglaucoma medication at the final index visit. Out of 20 patients, 18 were taking oral acetazolamide before treatment. Of these patients, 17 (94\%) did not require oral acetazolamide at the final visit. One patient who continued to use oral acetazolamide also had a higher IOP at the final index visit compared to pretreatment.

\section{Visual acuity (V/A) (Figure 3) (Table 1)}

Four patients (20\%) had V/A of $6 / 60$ Figure 3 or Table 1 better pretreatment. None of them deteriorated below $6 / 60$. There were no eyes with vision worse than perception of light (PL) at pretreatment. A total of 10 patients (50\%) lost vision; 10 patients $(50 \%)$ remained the same; and none improved.

\section{Retreatments}

Mean (SD) number of treatment sessions was 1.45 (0.68). All retreatments were performed within 18 months of the initial treatment session. Seven patients $(35 \%)$ had retreatments. These patients had better control of IOP ranging from 8 to $25 \mathrm{mmHg}$ at the final index visit and consequently were able to stop oral acetazolamide.

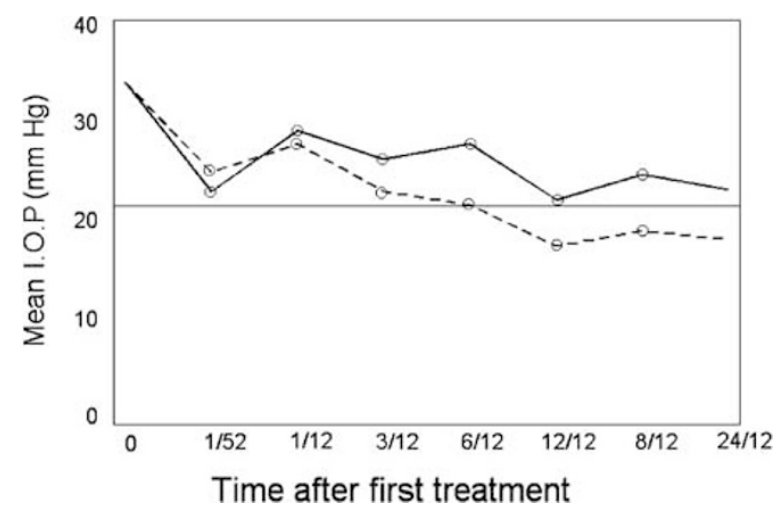

Figure 3 Graphical representation of deterioration of visual acuities. A total of 10 eyes (50\%) retained their original visual acuity. Numbers on the lines are indicative of number of eyes with a similar pattern of deterioration.

\section{Pars plana vitrectomy (Table 1)}

Out of 20 patients, 10 (50\%) had previous PPV Table 1. The indications for PPV were nonclearing vitreous haemorrhage or the presence of rubeosis iridis when the fundus could not be visualised owing to vitreous haemorrhage. PPV was performed on an urgent basis with an appropriate endolaser. Five out of 10 eyes had multiple PPVs. Two out of 10 patients had PPV for tractional retinal detachment involving the macula. Only one of these patients had required silicon oil, which was removed 4 weeks later because of raised IOP. 


\section{Hypotony and phthisis (Table 1)}

There were five eyes (25\%) with hypotony Table 1 (IOP $\leq 5)$. One of these eyes $(5 \%)$ became phthisical. All these eyes had two or more PPVs and only one treatment session that was performed on average 4.2 months (range of 3-6 months) after their last PPV. They developed hypotony approximately 3 months after treatment. One patient, who had vitreous haemorrhage and rubeosis iridis, developed a rhegmatogenous detachment 2 weeks after initial PPV. This was treated with further PPV, cryotherapy, encircling band, and silicon oil tamponade. Silicon oil was removed 4 weeks after surgery because of raised IOP. This patient had cyclodiode treatment 3 months after removal of silicon oil. This eye was noted to be phthisical at 1 year follow-up. All these five eyes were described as being comfortable at the last final index visit.

$\chi^{2}$ test $(P=0.001)$ suggests a significant relation between multiple PPVs and hypotony.

\section{Discussion}

There is a developing role for diode laser cyclophotocoagulation in the treatment of refractory glaucoma as a result of its published efficacy and its ease of use in an outpatient setting. It is portable and costeffective. It also appears to be safe.

There are relatively more studies in the literature that evaluate diode laser cyclophotocoagulation than other cyclodestructive modalities. Unfortunately, comparison of their results has been difficult because of two main factors. Firstly, the significant variation of treatment protocols used, even within the same study, and between studies; and, secondly, the extremely varied and usually complicated nature of underlying pathologies that lead to refractory glaucoma has made a 'like with like' comparison difficult. Retrospective studies ${ }^{10-13}$ evaluated $26-210$ patients with varied diagnoses. Laser power ranged from 1.5 to $2.5 \mathrm{~W}$ over $180-360^{\circ}$ of limbus. Some studies also adjusted the power for pops (a popping sound that reflects tissue disruption during the laser application). ${ }^{14}$ In 1999, Spencer and vernon ${ }^{9}$ suggested a standard protocol that worked well. This protocol was followed in this study. All of these had neovascular glaucoma secondary to diabetes. None of them had previous cyclodestructive or filtration surgery. Spencer and Vernon ${ }^{9}$ reported six rubeotic eyes out of 58 eyes recruited (diabetic and nondiabetic). Brancato et al ${ }^{5}$ reported a prospective, nonrandomised trial in 68 eyes, 10 of which were rubeotic. The rubeosis patients were not analysed separately in aforementioned studies. Walland ${ }^{16}$ treated 30 patients with rubeotic glaucoma; however, the amount of treatment was varied according to the clinical risk of phthisis.

The efficacy of treatment in the present study is comparable with other reported studies ${ }^{9,12}$ and is evidenced by $45 \%$ reduction in IOP and reduction in the amount of antiglaucoma medication required subsequently.

Being able to stop oral carbonic anhydrase inhibitor $\left(\right.$ Diamox ${ }^{\circledR}$ ) remains a desirable aim of any treatment in this group of patients and this was achieved in $94 \%$ of this series.

There were only four patients with measurable Snellen visual acuity. Two of these did not have any deterioration of vision during the course of study. The other two lost maximum of two Snellen visual acuity lines. From the remaining 16 patients, who had vision of counting fingers (CFs) or worse, eight deteriorated significantly. This could be the result of underlying progression of diabetic retinopathy and not necessarily the effect of the treatment. Spencer and Vernon ${ }^{9}$ also reported the poorest visual outcome in patients with diabetic retinopathy following treatment.

Our retreatment rate was $35 \%$, which is less than previous studies (Brancato et $a l^{5} 67 \%$, Block et al $70 \%$, and Spencer and Vernon ${ }^{9} 45 \%$ ). The retreatment rate for rubeotic eyes was not quoted in these studies. The patients in the present study who had retreatment had better control of IOP at the final index visit. This is in agreement with Spencer and Vernon. ${ }^{9}$

Five patients $(25 \%)$ developed hypotony one of which became phthisical. It is noticeable that all of these eyes had undergone multiple PPVs before the treatment. It is well recognised that panretinal photocoagulation performed during vitrectomy could result in limited destruction of ciliary body and hence reduction of IOP. ${ }^{18}$ If that was the case one would expect reduction of IOP following the PPV. No reduction of IOP was noted in these five eyes following PPV (cyclodiode treatment was performed on average 4.2 months after last PPV). These eyes only had one treatment and that was enough to induce hypotony. This is in contrast to previously held belief that diode laser cyclodestruction carries a low risk of phthisis and hypotony (Bloom et $a l^{2}$ reported hypotony in $0.6 \%$ of eyes receiving primary diode laser cyclodestruction). We believe that this does not hold true in neovascular glaucoma. It is already known that cyclocryotherapy (another commonly used cyclodestructive modality) carries $28 \%$ rate of hypotony ${ }^{19}$ and $12 \%{ }^{20,21}$ rate of phthisis which increases to $34 \%$ in the presence of neovascular glaucoma. ${ }^{22}$ This may be explained by the fact that the eyes with neovascular glaucoma have a disproportionately increased outflow resistance and their aqueous production is already compromised because of the underlying ischaemic 
process. These eyes probably do not have the same ability to autoregulate IOP as a normal eye. ${ }^{12}$ Any procedure such as cyclodestruction carries a significant risk of disturbing the balance between outflow resistance and aqueous production, which may result in hypotony.

This is the first study to analyse the outcome of treatment in a single category of patients with diabetic neovascular glaucoma. This approach may help future comparisons. We feel that the standard therapy regime as proposed by Spencer and Vernon ${ }^{9}$ needs to be modified for eyes with diabetic neovascular glaucoma that have had previous vitrectomy surgery. Owing to the risk of hypotony, vitrectomised eyes may require lower energy or fewer applications, probably the later, but this requires further investigation.

We did not find any study comparing augmented trabeculectomy with cyclodiode treatment. The risks of augmented trabeculectomy and tube surgery are well recognised. ${ }^{23-25}$ There is a need for a randomised study to evaluate these risks and compare them directly with cyclodiode treatment.

\section{Acknowledgement}

We thank the vitreo-retinal consultants, Dr T Barrie, Dr HH Hammer and Mr JR Murdoch for allowing us to use the clinical data on their patients.

\section{References}

1 Brown GC, Magargal LE, Schachat A, Shah H. Neovascualr glaucoma. Etiologic considerations. Ophthalmology 1984; 91: 315-320.

2 Sivak-Callcott JA, O'Day DM, Gass DM. Evidence based recommendations for the diagnosis and treatment of neovascular glaucoma. Ophthalmology 2001; 108: 1767-1778.

3 Ohnishi $Y$, Ishibashi T, sagawa T. Fluorescein gonioangiography in diabetic neovascularization. Graefes Arch Clin Exp Ophthalmol 1994; 232: 199-204.

4 Vogt A.Versuche zur intraokularen druckherabstzung mittelst diathermieschädigung des corpus ciliare (Zyklodiathermiestichelung). Klin Monatsbl Augenheilkd 1936; 97: 672-673.

5 Pastor SA, Singh K, Lee DA. Cyclophotocoagulation, a report by the American Academy of Ophthalmology. Ophthalmology 2001; 108: 2130-2138.

6 Hennis HL, Stewart WC. Semiconductor diode laser transscleral cyclophotocoagulation in patients with glaucoma. Am J Ophthalmol 1992; 113: 81-85.

7 Hawkins TA, Stewart WC. One year result of semiconductor trans-scleral cyclophotocoagulation in patients with refractory glaucoma. Arch Ophthalmol 1993; 111: 488-491.
8 Gaasterland DE, Pollack IP. Initial experience with a new method of trans-scleral cyclophotocoagulation for ciliary ablation in severe glaucoma. Trans Am Ophthalmol 1996; 103: 1294-1302.

9 Spencer AF, Vernon S. 'Cyclodiode' :result of a standard treatment. Br J Ophthalmol 1999; 83: 311-316.

10 Rebolleda G, Munoz FJ, Murube J. Audible pops during cyclodiode procedures. J Glaucoma 1999; 8: 177-183.

11 Ulbig MW, Mchugh DA, Mcnaught AI, Hamilton AM. Clinical comparison of semiconductor diode versus neodymium: YAG cyclophotocoagulation. $\mathrm{Br} J$ Ophthalmol 1995; 79: 569-574.

12 Bloom PA, Tsai JC, Sharma K, Miller MH, Rice NS, Hitchings RA et al. Cyclodiode. Trans-scleral diode laser cyclophotocoagulation in the treatment of advanced refractory glaucoma. Ophthalmology 1997; 104: 1508-1519; discussion 1519-1520.

13 Yap-Veloso MI, Simmons RB, Echelman DA, Gonzales TK, Veira WJ, Simmons RJ et al. Intraocular pressure control after contact trans-scleral diode cyclophotocoagulation in eyes with intractable glaucoma. J Glaucoma 1998; 7: 319-328.

14 Kosoko O, Gaasterland DE, Pollack IP, Enger CL. The diode laser ciliary ablation study group. Long-term outcome of initial ciliary ablation with contact diode laser cyclophotocoagulation for severe glaucoma. Ophthalmology 1996; 103: 1294-1302.

15 Brancato R, Carassa RG, Bettin P, Fiori M, Trabucchi G. Contact trans-scleral cyclophotocoagulation with diode laser in refractory glaucoma. Eur J Ophthalmol 1995; 5: 32-39.

16 Walland MJ. Diode laser cyclophotocoagulation: long term follow up of a standardized treatment protocol. Clin Exp Ophthamol 2000; 28(4): 263-267.

17 Block CJ, Freedman SF, Buckley EG, Shields MB. Transscleral diode laser cyclophotocoagulation for refractory pediatric glaucoma. J Pediatr Ophthalmol Strabismus 1997; 34: 235-239.

18 Vernon SA, Cheny H. Pan-retinal cryotherapy in neovascular disease. Br J Ophthalmol 1988; 72: 401-405.

19 Chee CK, Snead MP, Scott JD. Cyclocryotherapy for chronic glaucoma after vitreoretinal surgery. Eye 1994; 8: 414-418.

20 Benson MT, Nelson ME. Cyclocryotherapy: a review of cases over a 10 year period. Br J Ophthalmol 1996; 74: 103-105.

21 Brindley G, Sheilds MB. Value and limitations of cyclocryotherapy in neovascular glaucoma. Graefes Arch Clin Exp Ophthalmol 1986; 224: 545-548.

22 Krupin T, Mitchell KB, Becker B. Cyclocryotherapy in neovascular glaucoma. Am J Ophthalmol 1978; 86: 24-26.

23 Noureddin BN, Wilson-Holt N, Lavin $\mathrm{M}$ et al. Advanced uncontrolled glaucoma. Nd: YAG cyclophotocoagulation or tube surgery. Ophthalmology 1992; 99: 430-436.

24 Tsai JC, Feuer WJ, Parish II RK, Grajewski AL. 5-Fluorouracil filtering surgery and neovascular glaucoma. Long term follow up from original pilot study. Ophthalmology 1995; 102: 887-893.

25 Katz GJ, Higginbotham EJ, Lichter PR, Skuta GL, Musch $\mathrm{DC}$, Bergstrom TJ et al. Mitomycin C versus 5-fluorouracil in high risk glaucoma filtering surgery. Extended follow up. Ophthalmology 1995; 102: 1263-1269. 